\title{
A Review on Data Discrepancy Factor Performance for Industrial Applications using Clustering Algorithms
}

\author{
Indira Priyadarshini Tummala ${ }^{1, *}$ Ramesh $\mathrm{M}^{2}$ \\ ${ }^{1}$ CSE Department, Gokaraju Rangaraju Institute of Engineering \& Technology, Hyderabad, INDIA \\ ${ }^{2}$ CSE Department, Koneru Lakshmaiah Educational Foundation, Vaddeswaram, Guntur, INDIA
}

\begin{abstract}
DDF is the most significant measure among different bunch execution procedures to assess the immaculateness of any group component. Ordinarily, best groups are assessing by processing the quantity of information focuses inside a bunch. At the point when this tally is comparable to the quantity of required information focuses then this group is viewed as great. The greatness of the bunch system is fundamental not exclusively to discover the information check inside a group yet in addition to inspect it by totalling the information focuses these are (I) present inside a group where it ought not be and the other way around and (ii) not grouped for example anomalies (OL). The principle usefulness of DDF is that all bunch focuses can be gathered in comparative groups without exceptions, the current paper features on how contrasted with DDF more effective Clusters can be shaped through the Modern DDF. Further, we assess the exhibition of some grouping calculations, K-Means. As of late we, fostered the Modified K-Means Algorithm and Hierarchical Algorithm by utilizing the Data Discrepancy Factor (DDF).
\end{abstract}

\section{Introduction}

DDF is the main estimating strategy for the bunch execution The greatness of the group technique isn't to be settle relied upon just the information amount inward group, accordingly the adequacy of a bunches should be confirmed by totalling the information focuses these are (I) presently inside a group where it ought not be and the other way around and (ii) not bunched for example exceptions (OL). The standard handiness of DDF is all pack centers can be assembled in similar gatherings without special cases, but Cluster execution computation is the DDF assessment. It is enlisted by using standard formula given under. DDF is the essential assessment among any excess way to survey the show of any bundling in this paper appeared differently in relation to DDF the Modern DDF is more useful Clusters can be molded. The DDF can be process the how the gathering data centers will be planned in each bundle in $\mathrm{K}$ Means and Modified $\mathrm{K}$ Means and equivalently in different evened out gathering calculations [1].

Since the centre points are adaptable, the association topography might change rapidly and curiously as time goes on [8]. The organization is decentralized, where all organization action including finding the geography and conveying messages should be executed by the actual hubs, i.e., steering usefulness will be fused into portable hubs. The activity of practically every one of the calculations relies emphatically upon the introduction conditions. The arbitrary decision of the centroids is the principle downside for accomplishing a quick assembly and a worldwide least of the relating target work. The initial phase in current examination is the examination of the affectability of the calculations for fluctuating execution boundaries. For this investigation the chose informational collection incorporates just 365 day by day load bends for one year, to be specific year 2011 all through this Section. The $\mathrm{J}$ measure was utilized for the approval of all calculations since it addresses the most widely recognized quality trait of the arranged bunches, for example the union, which is communicated by the likenesses between the examples and the centroids. The $\mathrm{K}$-implies unites when the base measure of progress of the target work between two progressive emphases, as characterized by a preset resilience. The interaction is likewise ended after a pre-set most extreme number of emphases, in the event of no union. The principal test for the FCM intends to decide the proper worth of the remarkable boundary in (11), which controls the fluffiness of enrollment of each example. The variety of the boundary is with a stage of 0.10 . As the fluffiness file gets lower esteems, for example the produced segments are portrayed by higher vulnerability, the activity of the FCM turns out to be less productive. The impact of the fluffiness record on the grouping mistake turns out to be more apparent for huge number of bunches. After a bunch of investigations, the ideal worth of the file is acquired.

\section{Related Work}

Liu Xumin and Guan Yong grew new methodology of k-implies calculation is needed to keep not much information in all cycles and it is to be provided in the

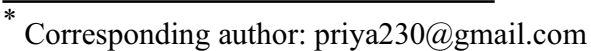


following emphasis. This calculation getting away from estimation of the distance of every information object to the bunch habitats over and over, compacting the execution time. Exploratory outcomes gave the improved calculation can effectively speed up bunching precision and diminishing the computational season of the k-implies [2]. Juanying Xie presented another methodology of K-implies bunching calculation, this grouping calculation giving powerful outcomes in mark of the square mean grouping mistake. It does not wager on any underlying qualities by testing the standard $\mathrm{K}$ implies calculation as a neighbourhood search system. It's everything except an orchestrated technique to pursue to apparent add one new bundle local area at each stage, but it similarly caused its generous computational weight. The principle advance in this computation is to finding the fundamental spot for the accompanying new gathering local area at each stage. This estimation moreover diminished its computational time [3]. In this paper we presented new sort of $\mathrm{K}$ Means Clustering calculation, contrasted with above calculations it run time is low and furthermore it improves bunch precision.

\section{Data Discrepancy Factor (DDF)}

It's everything except one more approach to manage notice the how the gathering data centers are occurred in each inside the pack, and it evaluate the show e of changed estimation of K-suggests, while accomplish the gathering on benchmark instructive list of SIS and h-g records. The two SIS, as like g-h records datasets, amazingly packed into their significant social affairs.
Their yields are shown in tables 1 and 2. Data Discrepancy Factor $\%=(\mathrm{AIn}+\mathrm{AOu}+\mathrm{OL}) / \mathrm{D}_{\mathrm{k}} * 100$

It was dictated by adding the hard and fast check of (I) "wrong data centers" occured inside bunch (AIn), (ii) the 'Right' data centers occurred outside of the bundle ( $\mathrm{AOu}$ ) of any one kth gathering and (iii) complete count of data centers, which are not to be assembled for instance the exemptions (OL) when found with the dispatch data (Dk). In the last computation, it is given as an irrelevant piece of the last count of data centers (N). Faultlessness of the DDF identical to $0 \%$, for instance it infers all the data centers are grouped effectively it's everything except a nil exemption. Moreover, other bundle execution is in like manner assessed same way using the DDF calculation. It is figured by using the condition given already. The DDF is fruitful expansion among any leftover quantum to assess the accomplishment of any packing methodology. Properly, the best batching is directed by registering the amount of things inside a gathering. In case the full scale objects composed to the amount of needed data centers, so those gathering are recognized to be extraordinary. The sufficiency of the clustering procedures need not be surveyed depending upon simply the data count inside a gathering, yet rather the reasonability of a gathering ought to be investigated by amassing up the data centers which are (I) current inside a pack where it should not be and the opposite way around and (ii) not grouped for instance oddities (OL). From tables 3 and 4, it infers that the changed computation of $\mathrm{K}$-infers gave in the current paper is performed well than normal [4].

Table 1. DDF computation on SIS data using algorithm K-mean

\begin{tabular}{|c|c|c|c|c|c|c|c|}
\hline \#Cluster & $\begin{array}{c}\text { Data } \\
\text { Points }\end{array}$ & Target & Observed & $\begin{array}{c}\text { Wrong } \\
\text { data points }\end{array}$ & OL & $\begin{array}{c}\text { Modern- } \\
\text { DDF (\%) }\end{array}$ & $\begin{array}{c}\text { Conventional } \\
\text { DDF (\%) }\end{array}$ \\
\hline 1 & $1-50$ & 50 & 61 & 14 & 0 & \multirow{2}{*}{$12 \%$} & \multirow{2}{*}{$16 \%$} \\
\cline { 1 - 6 } 2 & $51-100$ & 50 & 49 & 0 & 1 & $12 \%$ & \\
\hline 3 & $101-150$ & 50 & 39 & 3 & 0 & & \\
\hline
\end{tabular}

Table 2. DDF calculation on SIS dataset using modified K-Means algorithm

\begin{tabular}{|c|c|c|c|c|c|c|c|}
\hline \#Cluster & $\begin{array}{l}\text { Data } \\
\text { Points }\end{array}$ & Target & Observed & $\begin{array}{c}\text { Wrong } \\
\text { data points }\end{array}$ & OL & $\begin{array}{l}\text { Modern- } \\
\text { DDF (\%) }\end{array}$ & $\begin{array}{c}\text { Conventional } \\
\text { DDF (\%) }\end{array}$ \\
\hline 1 & $1-50$ & 50 & 49 & 0 & 1 & \multirow{3}{*}{$11.3 \%$} & \multirow{3}{*}{$17.3 \%$} \\
\hline 2 & $51-100$ & 50 & 62 & 14 & 0 & & \\
\hline 3 & $101-150$ & 50 & 38 & 2 & 0 & & \\
\hline
\end{tabular}

\section{Clustering Algorithms}

\subsection{K-Means Algorithm}

The primary burden profiling measure includes the choice and execution of at least one bunching calculations. The calculations ought to be executed for an alternate number of cycles and perhaps for a variable number of bunches. The following is a depiction of the grouping calculations utilized in this review. K-implies is the most famous parcel bunching calculation, because of its speed, adequacy and computational straightforwardness. In the normal K-implies the underlying centroids are picked haphazardly. After the choice of centroids, every one of the leftover examples is appointed to the nearest. The subsequent stage is the recomputation of the centroids, which are really the focuses of gravity of the recently arranged groups. The method is rehashed until there are no interpretations of examples between the groups. According to a numerical perspective the issue is to limit the accompanying target work. Customary K-means bunching calculation has a few downsides. The significant disadvantage of customary K-means grouping calculation is its presentation is predominantly relies upon the underlying centroids, which are chosen haphazardly and coming about bunches are distinctive for various runs for a 
similar information dataset. Another disadvantage incorporates distance computation cycle of customary kimplies calculation which requires some investment to merge bunching result, as it works out the separation from every information object to each group centroids in every emphasis while there is no compelling reason to ascertain that distance each time. As in the subsequent bunches a few information protests actually stays in a similar group after a few emphasis. It influences the exhibition of the calculations. Another disadvantage of $\mathrm{k}$-implies grouping is the necessity to give number of bunches shaped as contribution by the client. [5].

\subsection{Modified K Means Clustering Algorithm}

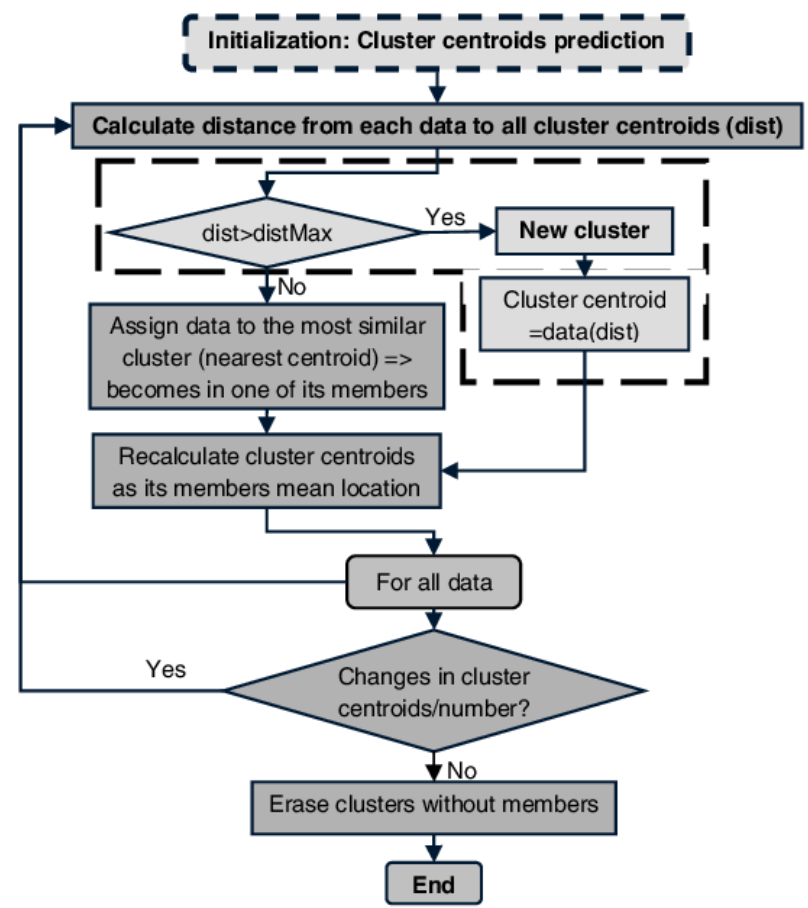

Fig. 1. Modified K-mean flowchart

\section{Results and Discussions}

Existing computation of K-suggests has been changed by introducing clustering and a while later reiterating $\mathrm{k}$ infers program to achieve all the nearer attributes of association. In the under graph is shown the customary Data Discrepancy factor for Clustering estimations on SIS, GHO and h-g records datasets [6]. The underneath diagram shows the Modern-DDF. Outline for these batching estimations on same datasets. Diverged from standard DDF, the Modern-DDF is great

\subsection{Accuracy of the K Means Clustering}

The beneath table shows the precision of the K Means calculation. This Algorithm is applied on Benchmark datasets GHO and SIS and furthermore my own informational index $\mathrm{H}$ and $\mathrm{G}$ Indices of the different creator's lists esteems up to 150 information records in each dataset [10].

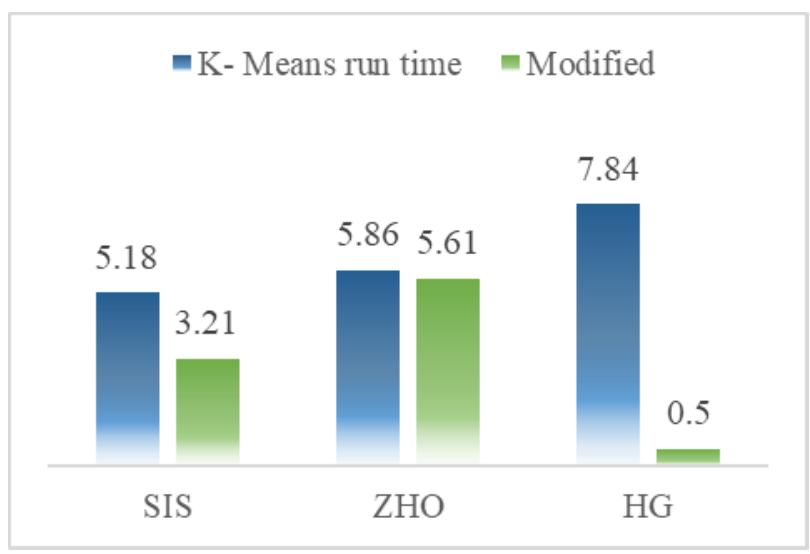

Fig. 2. Runtime Accuracy

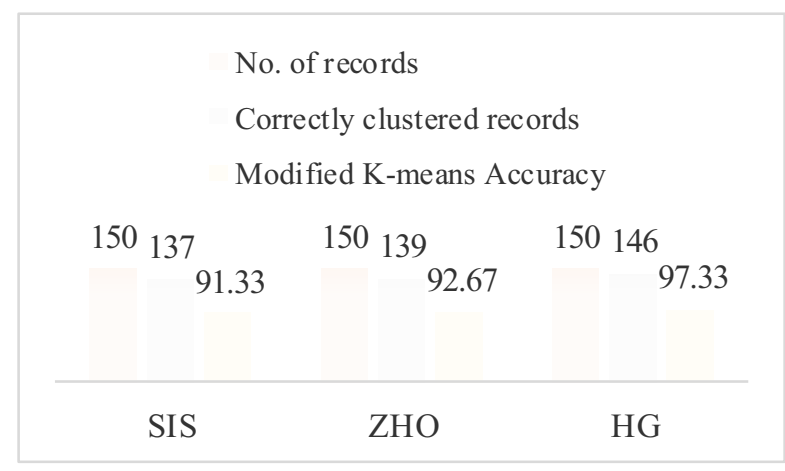

Fig. 3. DDF calculation for algorithms

\subsection{Accuracy of the Modified K Means Algorithm}

Contrasted with existing grouping calculations, adjusted k-implies calculation precision is the awesome. Existing estimation of $\mathrm{K}$-infers has been changed by introducing clustering and a while later repeating $\mathrm{k}$ suggests program to achieve all the nearer credits of association. The underneath layout shows the ModernDDF. Graph for these packing computations on same datasets. Appeared differently in relation to standard Exactness of bunching is dictated by contrasting the grouping results and the groups currently accessible in the UCI datasets [6]. Customary and further developed $\mathrm{k}$-implies grouping calculation gives diverse precision and time for each run as it chooses beginning centroid arbitrarily. So, these calculations are executed a few time and normal of exactness and time is taken. Exactness of proposed $\mathrm{k}$-implies bunching calculation is special at each run yet time is distinctive for each run, so it is likewise executed a few time and normal of time is taken. DDF, the Modern-DDF is great.

Table 3. Accuracy of Modified K-Means algorithm

\begin{tabular}{|c|c|c|c|}
\hline Database & $\begin{array}{l}\text { Modern- } \\
\text { DDF (\%) }\end{array}$ & $\begin{array}{c}\text { Conventional } \\
\text { DDF (\%) }\end{array}$ & Algorithm Used \\
\hline SIS & 12 & 16 & \multirow{2}{*}{ K-Means } \\
\hline GHO & 6 & 7.3 & \\
\hline H-G & 6.5 & 11 & \multirow{2}{*}{$\begin{array}{c}\text { Modified K- } \\
\text { Means }\end{array}$} \\
\hline SIS & 11.33 & 17.33 & 5.2 \\
\hline GHO & 4.5 & &
\end{tabular}




\begin{tabular}{|c|c|c|c|}
\hline H-G & 4.6 & 12.6 & \\
\hline SIS & 9.3 & 9.3 & Hierarchical \\
GHO & 7 & 7 & Agglomerative \\
Algorithm \\
\hline H-G & 0.66 & 0.6 & Algon \\
\hline
\end{tabular}

\subsection{Computational Time}

Achievement of the proposed calculation was assessed by figuring the computational time taken to do the run time utilizing SIS and h-g lists datasets. The outcomes are noted in tables beneath.

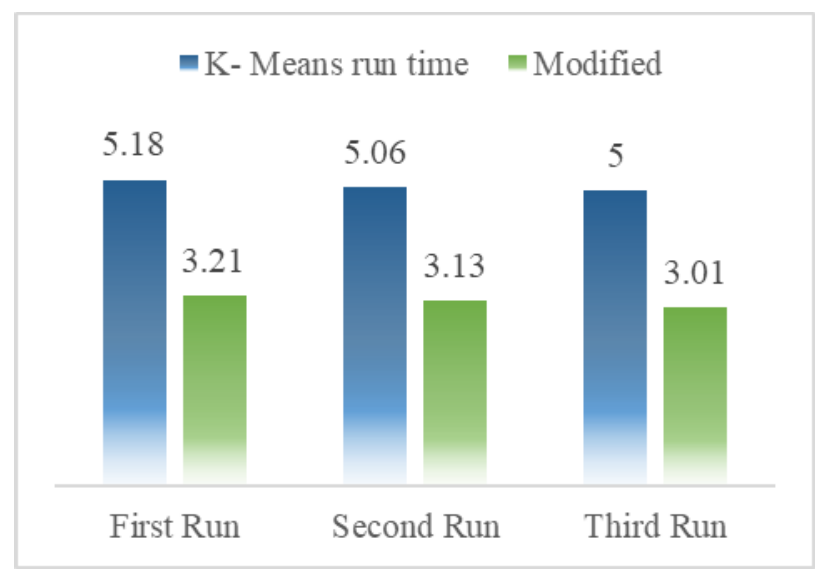

Fig. 4. Execution times for SIS dataset Runs.

The substance in table6 shows the quantity of bunches shaped in three datasets by utilizing grouping calculations. Examinations of the current K Means and Hierarchical agglomerative calculations and proposed KMean's calculation sets aside less effort to shape the different groups in given datasets [7]. The primary basic standard close to bunching examination is to arrange and parting informational index based on innate data related inside the aftereffect of such bunching measure is gathering of information focuses on an informational collection, where the items inside a gathering has an enormous arrangement of likeness and a low level of closeness with objects in different gatherings A critical field inside writing is references of articles distributed in different diaries. A creator could get reference to his paper once it contains data unwavering to the question of interest. As a general guideline, the more references a specific paper gets, the more noteworthy advantage is required to the distributer as far as legitimacy and specialized quality substance of the diary. This can be seen with the way that the subject picked by creators to distribute in one diary indicates the significance of observers to the diary [8]. The picked calculation depends upon the information need and the motivation behind bunching calculation. A portion of the calculations yield in bunches of same amounts while others make groups of unique size. A few calculations create circular groups while different calculation's structure stretched bunches, and a couple of bunching calculations are delicate to exceptions, etc. In any case, the yield is reliant upon how the information is preprepared and addressed [9].

Table 4. Computational time of algorithms

\begin{tabular}{|c|c|c|c|c|}
\hline Dataset & \# Clusters & $\begin{array}{c}\text { Execution Time } \\
\text { of K Means } \\
\text { Algorithm (sec) }\end{array}$ & $\begin{array}{c}\text { Execution Time of } \\
\text { Modified K Means } \\
\text { Algorithm (sec) } \\
\end{array}$ & $\begin{array}{c}\text { Hierarchical } \\
\text { Agglomerative } \\
\text { Algorithm } \\
\end{array}$ \\
\hline \multirow{5}{*}{ SIS } & 1 & 2.44 & 2.81 & \multirow{5}{*}{16.12} \\
\hline & 2 & 3.52 & 3.06 & \\
\hline & 3 & 6.63 & 3.30 & \\
\hline & 4 & 7.70 & 3.72 & \\
\hline & 5 & 8.84 & 4.11 & \\
\hline \multirow{5}{*}{$\mathrm{GHO}$} & 1 & 2.16 & 2.01 & \multirow{5}{*}{15.87} \\
\hline & 2 & 3.12 & 2.16 & \\
\hline & 3 & 3.98 & 2.76 & \\
\hline & 4 & 4.6 & 2.87 & \\
\hline & 5 & 6.2 & 3.0 & \\
\hline \multirow{5}{*}{$\mathrm{H}-\mathrm{G}$} & 1 & 2.10 & 0.12 & \multirow{5}{*}{15.33} \\
\hline & 2 & 6.16 & 0.16 & \\
\hline & 3 & 15.21 & 0.37 & \\
\hline & 4 & 16.20 & 0.39 & \\
\hline & 5 & 16.22 & 0.36 & \\
\hline
\end{tabular}

h-and g-file esteems informational index of creators who have distributed papers of logical fineness in diaries of notoriety. When mining and during data recovery the subject of value information with logical exceptional data and diary source is significant. The recovered data from a distributer source ought to be without mistake and hold a base number of references to the paper. Henceforth, information proportionality as for data and comparing creators alongside number of references are significant and consequently the context oriented bunching examination is introduced right now with legitimacy measurements on the adjusted calculation of $\mathrm{K}$-implies revealed somewhere else by our gathering. A few approval measures have been accounted for since numerous years, with ongoing technique expected each year, nonetheless, a few of the underlying calculations has demonstrated to be the generally proficient Validity measure is determined to figure out which is the most magnificent grouping by tracking down the base incentive for creators measure. Along these lines, the 
viable approvals conceivable with Davies-Bouldin file, Silhouette record and quantization blunder are introduced.

The underlying advance in calculation of K-implies is to isolate the given informational index into client characterized number of groups. The underlying choice of $\mathrm{k}$ in $\mathrm{k}$-implies is an interpretive choice and progressive runs ought to perform to acquire a streamlined division of information for any picked $\mathrm{k}$ worth. An earlier information on the information construction would bring about more suitable bunches. Nonetheless, as the information dimensionality upgrades, it turns out to be perpetually hard to choose a legitimate $\mathrm{K}$ worth. Subsequently, extensive consideration has been given to the subject of group approval, a technique which attempts to appraise a specific separating of information into bunches in request to think about legitimacy measurements for adjusted k-implies grouping; in this paper we utilized hand g-files informational index. The size and properties of the data set are differed from each other. The quantity of bunches went from 3 to 8 . The information was grouped utilizing the adjusted calculation of K-implies [10].

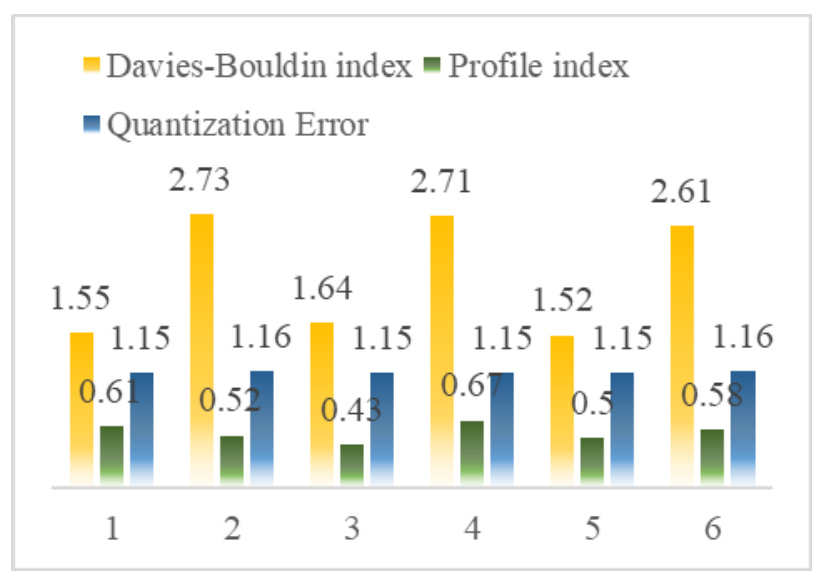

Fig. 5. Datasets vs index's and error

For the creators set of information, k-implies runs sequentially to make the best grouping for $\mathrm{k}$ upsides of the information somewhere in the range of 3 and 8 . These best bunching's were rehearsed by the three legitimacy strategies, bringing about a bunch of qualities for every legitimacy measure, one each for $\mathrm{k}=2$ through 8 . These scores were then contrasted against one another with track down the best $\mathrm{k}$ incentive for the grouping as per the legitimacy measure. The outcomes are given in the table [10]. From the outcomes it is seen that the redid calculation of $\mathrm{K}$-implies can create groups dependent on client input and the approval measurements announced here propose that the scores of every measurement with deference to k qualities are critical. The Davies-Bouldin list brought about a sensible number of groups and $\mathrm{k}=3$ addresses best bunch.

\section{Conclusion}

Examination of batching tells the proposed estimation of $\mathrm{K}$-infers is significantly more speed appeared differently in relation to the current estimation to the extent computational time and accuracy, bunch execution assessed by using the data irregularity factor. $\mathrm{K}$-implies bunching calculation is one of the most wellknown and a successful calculation to group datasets which is utilized in number of fields like logical and business applications. Nonetheless, this calculation has a few downsides, for example, choice of introductory centroid is irregular which doesn't ensure to yield extraordinary bunching result and k-implies grouping has more number of emphases and distance computations which at long last outcome in more measure of time to run. The Davies-Bouldin record, and Silhouette list perceived the right number of packs for all the $\mathrm{k}$ characteristics in the enlightening assortment.

\section{References}

1. S. Govinda Rao, and A. Govardhan. International Journal of Computer Applications 100.11 (2014).

2. Oyelade, O. J., O. Oladipupo, and I. C. Obagbuwa. arXiv preprint arXiv:1002.2425 (2010).

3. Bottegoni, Giovanni, et al. Bioinformatics 22.14 e58-e65, (2006)

4. Nagarjuna, A., sSuresh Kumar, T., Yogeswara Reddy Udaykiran, M. Int. Journal of Innovative Tech. Explo. Engg., 8, 640(2019)

5. Maulik, Ujjwal, and Sanghamitra Bandyopadhyay. IEEE Transactions on Pattern Analysis and Machine Intelligence24.12 1650-1654, (2002)

6. Xie, Juanying, et al. JCP 6.2 (2011): 271-279.on Pattern Analysis and Machine Intelligence 27.5 657-668, (2005).

7. Rao, J.S., Tummala, S.K., Kuthuri, N.R. Indo. Journal of Elect. Engg. and Computer Science, 21, 723 (2020)

8. Na, Shi, Liu Xumin, and Guan Yong. (IITSI), 2010 Third International Symposium on. IEEE, (2010).

9. Everitt, Brian S., et al. Cluster Analysis, 5th Edition 71-110, (2011)

10. Patel, Vaishali R., and Rupa G. Mehta. Computational Intelligence and Information Technology. Springer, Berlin, Heidelberg, 307-312, (2011). 